\title{
ON FINITE SIMPLE GROUPS WITH A SELF-CENTRALIZATION SYSTEM OF TYPE $(2(n))$
}

\author{
PAMELA A. FERGUSON
}

\begin{abstract}
Let $\boldsymbol{G}$ denote a simple group with a self-centralization system of type $(2(n))$, where $n>3$. Let $X_{1}$ denote an exceptional character of $G$, then $X_{1}(1)=k n+2 \varepsilon$ where $\varepsilon= \pm 1$. It is known that

$$
|G|=n X_{1}(1)\left(X_{1}(1)-\varepsilon\right)(\ln +1)
$$

where $l$ is a nonnegative integer. In this paper $G$ is classified if $l=0, \varepsilon=1$ and $X_{1}(1)$ is odd.
\end{abstract}

Let $G$ be a finite simple group, a proper subgroup $A$ of $G$ is called a $C C$ subgroup if $C_{G}(a) \subseteq A$ for all $a \in A^{\#}$. If $\left|N_{G}(A)\right| /|A|=2$ and $|A|=n$, then $G$ is said to have a self-centralization system of type $(2(n))$. The classification of simple groups with a self-centralization system of type $(2(n))$ is still incomplete. If $n=3$, then $G \cong \operatorname{PSL}(2, q), q=5$ or 7 [2]. If $n>3$, it is well known [5] that $G$ has $(n-1) / 2$ irreducible characters $X_{i}$, and one nonprincipal irreducible character $Y$ such that $X_{1}(1)=k n+2 \varepsilon, \quad Y(1)=k n+\varepsilon$ where $\varepsilon= \pm 1$ and $|G|=n X_{1}(1) Y(1)(\ln +1)$ where $l$ is a nonnegative integer. In all the known simple groups of type $(2(n)), l=0$ [5]. In this paper we classify all simple groups $G$ with a self-centralization system of type $(2(n))$ where $l=0, \varepsilon=1$, and $X_{1}(1)$ is odd. In particular we prove the following:

THEOREM A. Let $G$ be a finite simple group with a self-centralization system of type $(2(n))$ where $n \geqslant 5$. Let $A$ be a subgroup of order $n$ and let $X_{1}$ be an exceptional character of $G$ associated with $N(A)$. Assume $X_{1}(1)=k n+2$, $X_{1}(1)$ is odd and $|G|=n(k n+2)(k n+1)$, then $G$ is isomorphic to $\operatorname{Sz}(q)$ or $\operatorname{PSL}\left(2,2^{r}\right)$.

Let $G \in$ Hypothesis A if $G$ satisfies the hypothesis of Theorem A but not the conclusion. Let $\tau$ be an involution in $N(A)$, Theorem 5.1 [5] implies $G$ has one class of involutions. If $S$ is a set, let $|S|$ denote the number of elements in $S$.

Assume $G \in$ Hypothesis A.

If $\left|C_{G}(\tau)\right|=2^{r}$, then [6] implies either $G$ satisfies Theorem $A, G \cong$ $\operatorname{PSL}(3,4)$ or $G \cong \operatorname{PSL}(2, q)$ where $q$ is odd, $n=(q+1) / 2$ or $(q-1) / 2$ where $n$ is odd. However $X_{1}(1)$ is even for $\operatorname{PSL}(3,4)$ or $\operatorname{PSL}(2, q), q$ odd. Let $G_{2}$ be a Sylow 2 subgroup of $G$; if $G_{2}$ is abelian, then [7] again implies a

Received by the editors January 26, 1978.

AMS (MOS) subject classifications (1970). Primary $20 \mathrm{Cl} 5$. 
contradiction. Hence, $G \in$ Hypothesis A implies $\left|C_{G}(\tau)\right| \neq\left|G_{2}\right|$ and $G_{2}$ is not abelian.

If $z \in C_{G}(\tau)^{\#}$, let $F_{z}=\left\{\tau^{g} \mid \tau^{g} \in C_{G}(z)\right\}$.

Lemma 1. Assume $G \in$ Hypothesis A; then there is an element $x \in C_{G}(\tau)^{\sharp}$ such that $x$ has odd order and $F_{x} \neq F_{\tau}$.

Proof. Assume $x$ an element of odd order in $C_{G}(\tau)^{\#}$ implies $F_{x}=F_{\tau}$. $G \in$ Hypothesis A implies $\left|F_{\tau}\right| \neq 1$. Let $\tau_{2} \in F_{\tau}-\{\tau\}$; then $\tau_{2}=\tau^{g}$ for some $g \in G$. Now $\tau_{2} \in F_{x}$ implies $x \in C_{G}\left(\tau_{2}\right)=\left(C_{G}(\tau)\right)^{g}$. Hence $x=y^{g}$ where $y$ has odd order and $y \in C_{G}(\tau)^{\#}$. Therefore $F_{x}=F_{y} g=\left(F_{y}\right)^{g}=\left(F_{\tau}\right)^{g}$ $=F_{\tau_{2}}$. Hence $F_{\tau}=F_{\tau_{2}}$. Let $\left\langle F_{\tau}\right\rangle$ be the group generated by $F_{\tau}$, then $F_{\tau_{2}}=F_{\tau}$ implies $\left\langle F_{\tau}\right\rangle$ is an abelian 2-group. Thus $\Omega_{1}\left(G_{2}\right)$ is abelian and Goldschmidt [3] implies $G \notin$ Hypothesis A.

Proof of Theorem A. We will assume $G \in$ Hypothesis A and obtain a contradiction. Let $Y$ be the nonprincipal nonexceptional character associated with $N(A)$. Since $\left|G_{2}\right| \mid Y(1), Y(\tau)=0$ and Lemma 6 of [4] implies $\left|C_{G}(\tau)\right|=$ $Y(1)$. Theorem $17.4[1]$ implies $Y(z)=0$ for $z \in C_{G}(\tau)^{\sharp}$. Since $\varepsilon=1$, Lemma 4 [4] implies $X_{i}(z)=1$ for $z \in C_{G}(\tau)^{\#}$, and $i=1, \ldots,(n-1) / 2=t$.

Let $T$ denote the principal character of $C_{G}(\tau)$ and let $T^{*}$ denote the character of $G$ induced by $T$. Let $1_{G}$ denote the principal character of $G$. Frobenius Reciprocity now implies $\left(T^{*}, X_{i}\right)=2$ for $i=1, \ldots, t$, and $\left(T^{*}, Y\right)=\left(T^{*}, 1_{G}\right)=1$. Now $T^{*}(1)=|G| / Y(1)=n X_{1}(1)$ and $\sum_{i=2}^{t} 2 X_{i}(1)+$ $Y(1)+1_{G}(1)=n X_{1}(1)$ imply $T^{*}=\sum_{i=1}^{t} 2 X_{i}+Y+1_{G}$. Hence, $z \in C_{G}(\tau)^{\sharp}$ implies $T^{*}(z)=n$.

If $z \in C_{G}(\tau)^{\#}$, then $T^{*}(z)$ is the number of involutions in $C_{G}(z)$; hence $\left|F_{z}\right|=n$. Let $x \in C_{G}(\tau)^{\#}$ where $x$ has odd order and let $z=x \tau$. Now $F_{z}=F_{x} \cap F_{\tau}$. Hence $\left|F_{z}\right|=n=\left|F_{\tau}\right|=\left|F_{x}\right|$ implies $F_{\tau}=F_{z}=F_{x}$. This contradicts Lemma 1.

\section{REFERENCES}

1. W. Feit, Characters of finite groups, Benjamin, New York, 1967.

2. W. Feit and J. G. Thompson, Finite groups which contain a self-centralizing subgroup of order 3, Nagoya Math. J. 21 (1962), 185-197.

3. D. Goldschmidt, 2-fusion in finite groups, Ann. of Math. (2) 99 (1974), 70-117.

4. K. Harada, $A$ characterization of groups $F(2, q)$, Illinois J. Math. 11 (1967), 647-659.

5. G. Higman, Odd characterizations of finite simple groups, Lecture Notes, Univ. Michigan, Ann Arbor, Mich., 1968.

6. M. Suzuki, Finite groups with nilpotent centralizers, Trans. Amer. Math. Soc. 99 (1961), 425-470.

7. J. Walther, The characterization of finite groups with abelian 2-subgroups, Ann. of Math. (2) 89 (1969), 405-514.

Department of Mathematics, University of Miami, Coral Gables, Florida 33124 